\title{
TRIM21-regulated Annexin A2 plasma membrane trafficking facilitates osteosarcoma cell differentiation through the TFEB-mediated autophagy
}

Huan-Tian Zhang ${ }^{1,2}$, Qingzhong Zeng ${ }^{1}$, Baomeng Wư ${ }^{1}$, Junlei Lu', Kui-Leung Tong ${ }^{2}$, Jiebin Lin², Qiu-Yu Liư ${ }^{3}$ Lipei Xu',

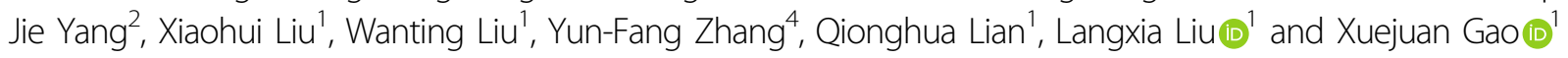

\begin{abstract}
Osteosarcoma (OS) is the most common primary malignant bone tumor in children and adolescents, which is characterized by dysfunctional autophagy and poor differentiation. Our recent studies have suggested that the tripartite motif containing-21 (TRIM21) plays a crucial role in regulating OS cell senescence and proliferation via interactions with several proteins. Yet, its implication in autophagy and differentiation in OS is largely unknown. In the present study, we first showed that TRIM21 could promote OS cell autophagy, as determined by the accumulation of LC3-II, and the degradation of cargo receptor p62. Further, we were able to identify that Annexin A2 (ANXA2), as a novel interacting partner of TRIM21, was critical for TIRM21-induced OS cell autophagy. Although TRIM21 had a negligible effect on the mRNA and protein expressions of ANXA2, we did find that TRIM21 facilitated the translocation of ANXA2 toward plasma membrane (PM) in OS cells through a manner relying on TRIM21-mediated cell autophagy. This functional link has been confirmed by observing a nice co-expression of TRIM21 and ANXA2 (at the PM) in the OS tissues. Mechanistically, we demonstrated that TRIM21, via facilitating the ANXA2 trafficking at the PM, enabled to release the transcription factor EB (TFEB, a master regulator of autophagy) from the ANXA2-TFEB complex, which in turn entered into the nucleus for the regulation of OS cell autophagy. In accord with previous findings that autophagy plays a critical role in the control of differentiation, we also demonstrated that autophagy inhibited OS cell differentiation, and that the TRIM21/ANXA2/ TFEB axis is implicated in OS cell differentiation through the coordination with autophagy. Taken together, our results suggest that the TRIM21/ANXA2/TFEB axis is involved in OS cell autophagy and subsequent differentiation, indicating that targeting this signaling axis might lead to a new clue for OS treatment.
\end{abstract}

Correspondence: Langxia Liu (tliulx@jnu.edu.cn) or

Xuejuan Gao (tgaoxj@jnu.edu.cn)

${ }^{1}$ Key Laboratory of Functional Protein Research of Guangdong Higher Education Institutes and MOE Key Laboratory of Tumor Molecular Biology, Institute of Life and Health Engineering, Jinan University, Guangzhou 510632, China

${ }^{2}$ Department of Bone and Joint Surgery, Institute of Orthopedic Diseases, The First Affiliated Hospital, Jinan University, Guangzhou, Guangdong 510630, China

Full list of author information is available at the end of the article

These authors contributed equally: Huan-Tian Zhang, Qingzhong Zeng, Baomeng Wu

Edited by G. M. Fimia

\section{Introduction}

Osteosarcoma (OS) is the most common primary bone malignancy in children and adolescents ${ }^{1}$. Although the survival rate of patients with a localized tumor can reach $70 \%$ at 5 years, for those who developed pulmonary metastatic or relapsed, its survivorship is significantly reduced to only $20-30 \%^{2,3}$. This poor clinical outcome underscores the urgent need to clarify the mechanism of OS pathogenesis aiming to develop novel therapeutic strategies. Increasing evidence has suggested that more than $80 \%$ of OS are poorly differentiated and a better-differentiated state is associated

\section{(c) The Author(s) 2021}

(c) (i) Open Access This article is licensed under a Creative Commons Attribution 4.0 International License, which permits use, sharing, adaptation, distribution and reproduction c. in any medium or format, as long as you give appropriate credit to the original author(s) and the source, provide a link to the Creative Commons license, and indicate if changes were made. The images or other third party material in this article are included in the article's Creative Commons license, unless indicated otherwise in a credit line to the material. If material is not included in the article's Creative Commons license and your intended use is not permitted by statutory regulation or exceeds the permitted use, you will need to obtain permission directly from the copyright holder. To view a copy of this license, visit http://creativecommons.org/licenses/by/4.0/. 
with a better prognosis of the patients ${ }^{4,5}$. Thus, exploring the molecular mechanism underlying differentiation defects in OS is insightful for OS therapy ${ }^{4,5}$.

The tripartite motif proteins (TRIM) have increasingly been recognized to play a role in regulating autophagy and are implicated in innate immune responses ${ }^{6}$. Among them, TRIM21 assembles autophagy machinery through interacting with several key components of the autophagic machinery and acts as an autophagic receptor ${ }^{7}$; whereas, its implication in tumor autophagy has not been investigated. Recent reports have shown that TRIM21 participates in the regulation of immune cell subset differentiation ${ }^{8,9}$; however, its effect on tumor cell differentiation has not been uncovered. A series of studies have highlighted the role of TRIM21 in multiple cancers. For instance, TRIM21 has been demonstrated to be downregulated in hepatocellular carcinoma, breast cancer, and diffuse large B-cell lymphomas ${ }^{10-13}$, whereas other studies have shown that decreased expression of TRIM21 predicts better prognosis in pancreatic cancer patients ${ }^{14}$. We have recently demonstrated that TRIM21 plays a crucial role in regulating OS cell senescence and proliferation via interacting with several proteins, such as PRMT5 ${ }^{15,16}$. However, it remains to be investigated whether TRIM21 plays a role in regulating OS autophagy and subsequent differentiation.

In this study, we first demonstrated that TRIM21 played a crucial role in regulating OS autophagy. Mechanistically, we found that TRIM21, via interacting with Annexin A2 (ANXA2), regulated its plasma membrane (PM) localization, which in turn, facilitated the nuclear translocation of transcription factor EB (TFEB). Altogether, we propose that the TRIM21/ANXA2/TFEB axis regulates OS cell differentiation via the induction of autophagy.

\section{Materials and methods \\ Antibodies and reagents}

The following antibodies were obtained from Cell Signaling Technology (USA): p62 (\#23214), Beclin 1 (\#3495), LAMP1 (\#9091), HistonH3 (\#4499), atp A1 (\#96292), GFP (\#2555), and LC3-II (\#2775). Anti-TRIM21 (sc-25351) was from Santa Cruz Biotechnology (USA). Antibodies against RUNX2 (ab23981) and TFEB (ab220695) were from Abcam (USA). Antibodies against ANXA2 (11256-1-AP, 66035-1lg), CD63 (25682-1-AP), $\beta$-actin (20536-1-AP), $\beta$-tubulin (10094-1-AP), and GAPDH (10494-1-AP) were from Proteintech (USA). Antibodies against LC3 (L7543), Flag (SAB4301135), and HA (H6908) were from Sigma (USA). Alexa Fluor 488- and 594-conjugated secondary antibodies (ZF-0511, ZF-0512, ZF-0516, ZF-0513) were from ZSGBBIO (China). Chloroquine phosphate (CQ, PHR1258-1G), tetracycline (TET, \#58346), 3-MA (M9281), MG132 (\#474790), rapamycin (RAPA) (V900930-1mg), and DAPI (\#10236276001) were from Sigma (USA).

\section{Cell lines and culture}

Human OS U2-OS and Saos-2 cells (ATCC, USA) were maintained in McCoy's 5A medium (Sigma, USA) containing $10 \%$ or $15 \%$ fetal bovine serum (FBS, PAN-Biotech, Germany) in a humidified atmosphere containing $5 \% \mathrm{CO}_{2}$. MG63 cells (Institute of Life Science Chinese Academy of Sciences, China) were cultured in MEM (Gibco BRL, USA) and supported with 10\% FBS. All these cells were authenticated by short tandem repeat (STR) as described previously ${ }^{17}$ and were mycoplasma-free tested by TransDetect ${ }^{\circledR}$ PCR Mycoplasma Detection Kit (TRANS, China).

\section{Small interfering RNA transfection}

Sequences of the small interfering RNAs (siRNAs) used in this study were listed as follows: 5'-GCAGGAGUUGGCU GAGAAGTT-3' (TRIM21), 5'-GGGUCUGUCAAAGCCU AUATT-3' (ANXA2), 5'-CAGTTTGGCACAATCAATA TT-3' (Beclin 1), 5'-AGACGAAGGUUCAACAUCA-3' (TFEB), and 5'-UUCUCCGAACGUGUCACGUTT- $3^{\prime}$ (NC). OS cells were transfected with the siRNAs using Lipofectamine ${ }^{\mathrm{TM}} 2000$ (Invitrogen, CA, USA) according to the manufacturer's instructions.

\section{Establishment of OS stable cells}

The U2-OS cells stably expressing H125-TRIM21 or H125-V were established previously and the MG63 stable cells were established using the same method $^{15}$. U2-OS cells stably knocking down TRIM21 and its control cells were named as sh-TRIM21\#1, sh-TRIM21\#2, and sh-NC as previously described ${ }^{16}$.

\section{Co-immunoprecipitation}

The co-immunoprecipitation (co-IP) assay was performed as described previously ${ }^{18}$. Briefly, $1 \mathrm{mg}$ of total protein from the lysate was mixed with $2 \mu \mathrm{g}$ of indicated primary antibodies. The immune complexes were separated by western blotting. These experiments were repeated three times.

\section{Immunofluorescence assays}

Immunofluorescence (IF) assay was performed to determine the subcellular localization of the indicated proteins as described previously ${ }^{19,20}$. To observe the subcellular localization of GFP-ANXA2 in living cells, the cells cultured on coverslip-bottomed small chamber were transfected with GFP-ANXA2 and then mounted onto the stage of LSM700 microscope equipped with a temperature-controlled and $\mathrm{CO}_{2}$-controlled small incubator as described previously ${ }^{21}$.

\section{ELISA kit for alkaline phosphatase}

Cultured cells were lysed with lysis buffer A (20 mM Tris $\mathrm{pH} 7.5,150 \mathrm{mM} \mathrm{NaCl}, 1 \%$ Triton X-100, sodium pyrophosphate, $\beta$-glycerophosphate, EDTA, $\mathrm{Na}_{3} \mathrm{VO}_{4}$, leupeptin, and $1 \%$ protease inhibitor cocktail (Roche)). Alkaline 
phosphatase (ALP) activity of the lysates (50 ng/well) was examined by ELISA kit (YEASEN, China) according to the manufacturer's protocol. Absorbance at $450 \mathrm{~nm}$ was measured using a spectrophotometric microplate reader (ELX800, BioTek Instrument, USA). These experiments were repeated three times.

\section{Gene expression by qRT-PCR}

Total RNA was purified and the quantitative real-time PCR (qRT-PCR) assays were performed as described previously $^{19}$. The following primers were used: TRIM21, 5' - TGGACAATTTGGTTGTGGAA-3' (forward), 5' -ACC ATGCCAGCCTCATAGTC-3' (reverse); ALP, 5'-GACA AGAAGCCCTTCACTGC-3' (forward), 5'-AGACTGC GCCTGGTAGTTGT-3' (reverse); RUNX2, 5'-CGGAAT GCCTCTGCTGTTAT-3' (forward), 5'-TGGGGAGGAT TTGTGAAGAC-3' (reverse); ANXA2, 5'-CAGGATAT TGCCTTCGCCTACCAG-3' (forward), 5'-GCGTCATA CTGAGCAGGTGTCTTC-3' (reverse); p62, 5'-GCCTC TGGTTCTGACACTTT-3' (forward), 5'-GGTGAGGTG GAAGGCATTTA-3' (reverse); ACTB (the control), 5'ACGTGGACATCCGCAAAG-3' (forward), 5'-GACTC GTCATACTCCTGCTTG-3' (reverse). These experiments were repeated three times.

\section{Subcellular fractionation assay}

For the preparation of nuclear, cytoplasmic, and membrane-enriched proteins, Minute ${ }^{\mathrm{TM}}$ Plasma Membrane Protein Isolation and Cell Fractionation Kit (SM-005, Invent Biotechnologies, Eden Prairie, USA) was applied according to the manufacturer's instructions. Then, the western blotting assay was used to analyze the protein expression in different fractionations. These experiments were performed three times.

\section{Western blotting}

Western blotting was performed as described previously $^{18,19}$. Three independent experiments were performed and the relative quantitative expression was analyzed by Image J software (NIH, USA).

\section{Tissue microarrays}

The expressions of TRIM21 and ANXA2 were analyzed using two consecutively numbered commercial tissue microarrays (TMAs) of OS (OS804C, Alenabio, Xi'an, China), which includes 80 sections of OS tissue samples and every 2 sections coming from one OS case. The company stated that all human tissues are collected under Institutional Review Board and Health Insurance Portability and Accountability Act (HIPAA)-approved protocols. The immunohistochemical staining was performed in Shanghai Outdo Biotech (China) as described previously $^{18}$. Two slides were stained with the primary antibodies against TRIM21 (1:200 dilution) and ANXA2
(1:500 dilution). Histologic slides were reviewed by two experienced pathologists without knowledge of the relevant information of the patients as reported previously ${ }^{18}$. Briefly, the intensity of protein staining was scored as 0 (negative), 1 (weak), 2 (moderate), 3 (stronger), or 4 (strongest). The percentage of positive cells was scored as 0 (<10\%), 1 (11-40\%), 2 (41-60\%), 3 (61-80\%), and 4 $(>80 \%)$. These two scores were then multiplied. Correlation between the expression of TRIM21 and ANXA2 was estimated using Spearman's rank correlation analysis.

\section{Statistical analysis}

All values were shown as the means \pm SEM of three independent experiments. Statistical analyses were calculated using Student's $t$-test and the statistical significance was defined as $p<0.05$.

\section{Results}

\section{TRIM21 induces autophagy of OS cells}

TRIM21 has been reported to be involved in regulating autophagy of immune cells ${ }^{7}$, yet its role in OS autophagy is largely unknown. We first determined whether TRIM21 regulated the expression of LC3-II, an autophagy marker of autophagosomes ${ }^{3}$. As shown in Fig. 1A, B, overexpression of HA-TRIM21 promoted the expression of LC3-II. Autophagy inhibitor CQ causes LC3-II accumulation, due to its prevention of autophagosome-lysosome fusion as reported previously ${ }^{22}$. TRIM21 overexpression caused a further increase of CQ-mediated ( $5 \mu \mathrm{M})$ LC3-II accumulation. In contrast, knockdown of TRIM21 reduced the accumulation of LC3-II in both the control and CQstimulated conditions (Fig. 1C, D). Next, IF assay using a $\mathrm{pH}$-sensitive mCherry-EGFP-LC3 ${ }^{23}$ also showed that overexpression of TRIM21 remarkably increased the ratio of red-only to yellow dots, whereas knockdown of TRIM21 attenuated this ratio (Fig. 1E), suggesting that TRIM21 is essential for promoting the formation of autophagosomes and its delivery to lysosomes.

Then, we tested whether TRIM21 participated in the degradation of $\mathrm{p} 62$, an autophagy receptor, which can be degraded together with cargos $^{3,24}$. Overexpression of TRIM21 significantly diminished the p62 expression, whereas, its expression level was restored by CQ (Fig. 1F). In contrast, TRIM21 knockdown inhibited the degradation of p62, similar to the effect of CQ (Fig. 1D). These results collectively suggest that TRIM21 plays a crucial role in the induction of OS cell autophagy.

\section{ANXA2 is identified as a novel interacting protein of TRIM21, and it counteracts on TRIM21-mediated autophagy}

Next, we sought to explore the underlying mechanism of TRIM21-mediated OS cell autophagy. Previously, we had identified a series of interacting proteins of TRIM21 


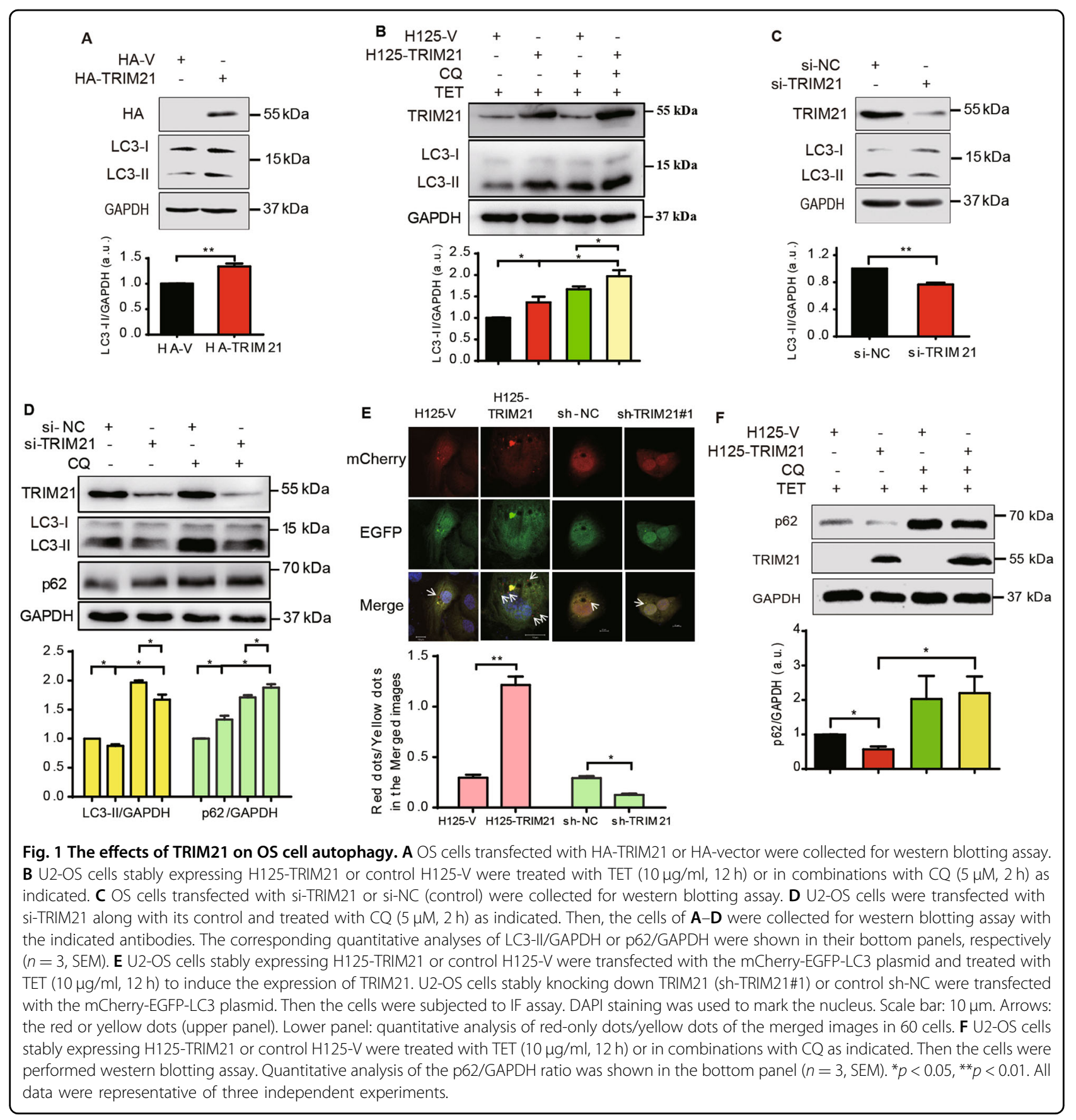

in the U2-OS cells ${ }^{15}$. Among them, ANXA2 exhibited the highest unique peptide number and sequence coverage rate (Supplementary Table S1). ANXA2 is a calcium- and phospholipid-dependent membrane-binding protein that has been reported to regulate osteogenic differentiation, autophagy, and OS tumorigenesis ${ }^{25-28}$; we thus hypothesized that ANXA2 play a role in TRIM21-regulated autophagy via protein-protein interaction. Notably, a cytoplasmic colocalization of TRIM21 and ANXA2 was observed in U2-OS cells, with a marked decrease when TRIM21 was knocked down (Fig. 2A). Co-IP assay further validated that ANXA2 was precipitated by the HA antibody in the HA-TRIM21 group but not in control cells (Fig. 2B). Similarly, endogenous TRIM21 and ANXA2 were coprecipitated by the ANXA2 antibody (Fig. 2C), suggesting that TRIM21 and ANXA2 interacted with each other. Interestingly, another autophagy-related protein, Beclin 1, was also identified in the same immune complex of HATRIM21 (Fig. 2B), indicating that TRIM21 might also 


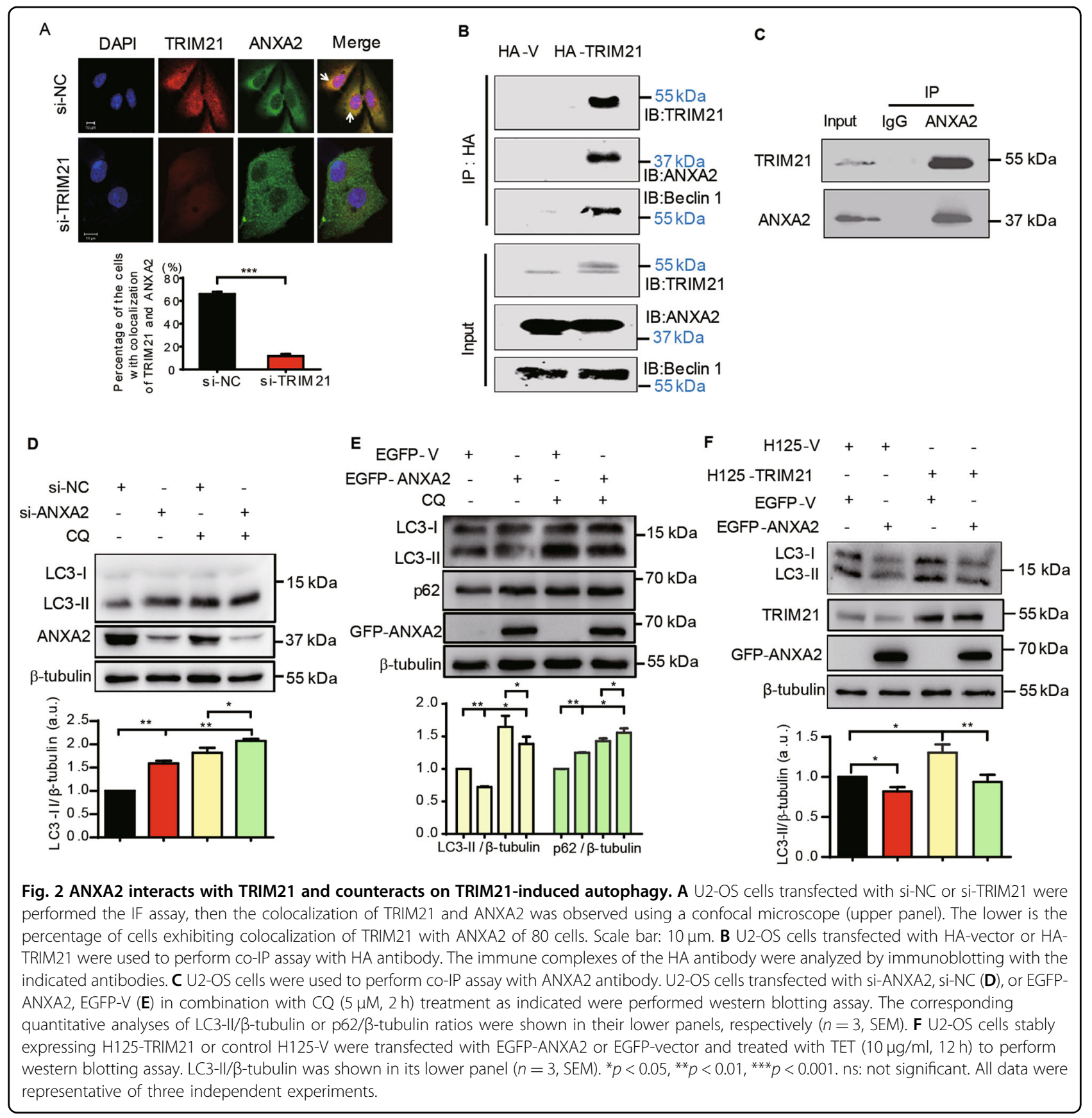

participate in the early stage of autophagy through interacting with Beclin 1. Next, we explored the role of ANXA2 in TRIM21-mediated OS cells autophagy. ANXA2 knockdown significantly increased the LC3-II expression either in the presence or absence of $\mathrm{CQ}$ (Fig. 2D). Conversely, overexpression of EGFP-ANXA2 reduced the expression of LC3-II, while increased the accumulation of p62, which was also observed when combined with CQ treatment (Fig. 2E). Furthermore, we did find that overexpression of ANXA2 compromised the induction of LC3-II by enforced TRIM21 expression
(Fig. 2F). These results together suggest that ANXA2 negatively regulates OS cell autophagy induced by TRIM21.

TRIM21 promotes the translocation of ANXA2 toward cell membrane

Next, we investigate whether TRIM21, as an E3 ubiquitin ligase $^{29}$, may regulate the expression of ANXA2. Our results showed that neither overexpression nor knockdown of TRIM21 had visible effects on the protein and mRNA expression of ANXA2 (Supplementary Fig. S1). As the subcellular trafficking of ANXA2 is another 

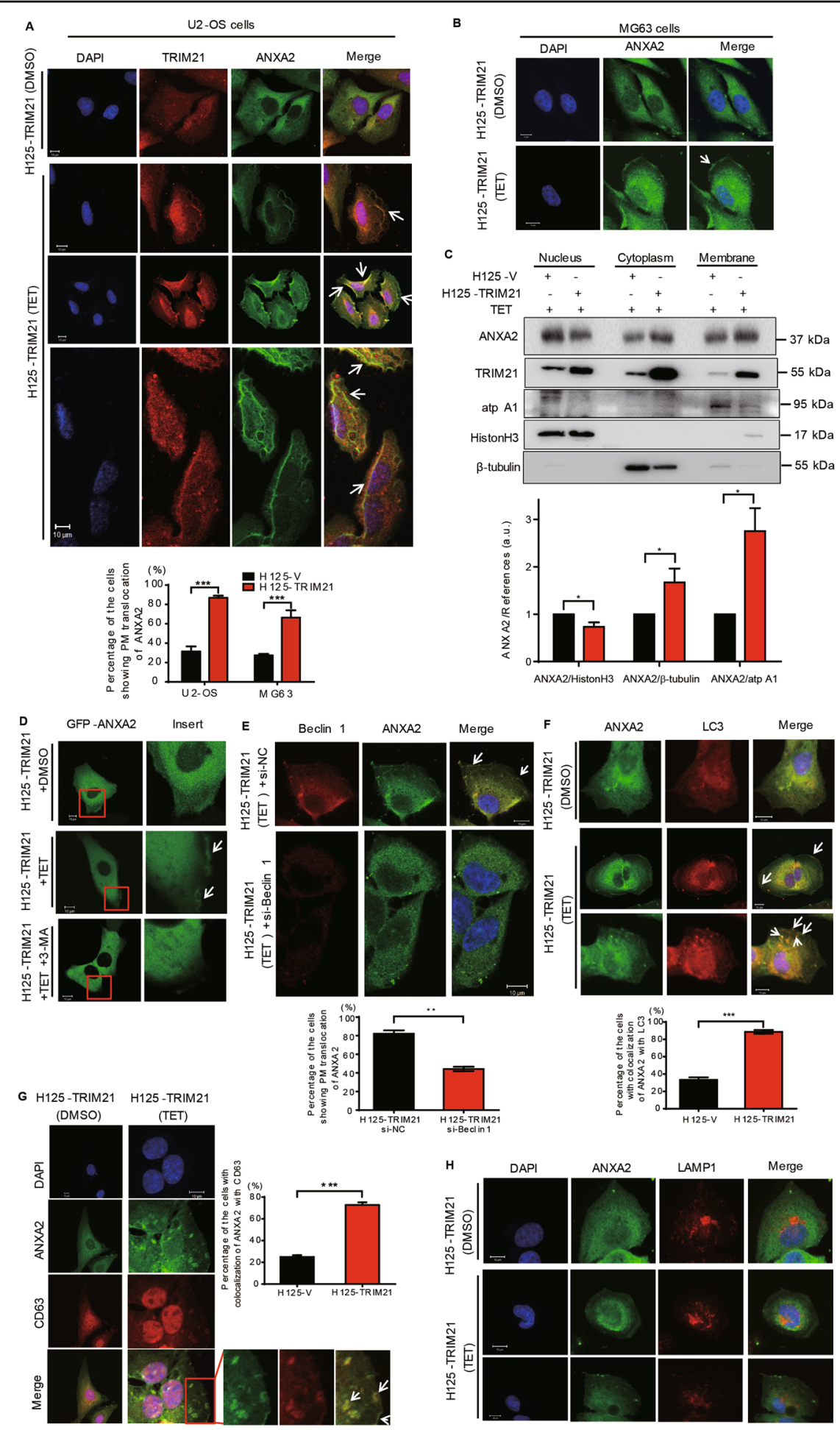

Fig. 3 (See legend on next page.)

major form of ANXA2 regulation, we then tested whether TRIM21 plays a role in regulating its localization. As shown in Fig. 3A, B, stable overexpression of TRIM21 remarkably increased the translocation of ANXA2 toward PM in both the U2-OS and MG63 cells. Consistently, the subcellular fractionation assay also demonstrated that 
(see figure on previous page)

Fig. 3 TRIM21 promotes the translocation of ANXA2 toward PM. A U2-OS cells stably expressing H125-TRIM21 were treated with DMSO or TET $(10 \mathrm{\mu g} / \mathrm{ml})$ to induce the overexpression of TRIM21 and then carried out IF assay (upper panel). Percentage of the cells showing PM translocation of ANXA2 of 90 cells in $\mathbf{A}$ and $\mathbf{B}$ (lower panel). B MG63 cells stably expressing H125-TRIM21 were treated with DMSO or TET (10 $\mu \mathrm{g} / \mathrm{ml}$ ) and then carried out IF assay. C U2-OS cells stably expressing H125-TRIM21 or H125-V were treated with TET $(10 \mu \mathrm{g} / \mathrm{ml})$ and then performed subcellular fractionation assay to isolate the nuclear, cytoplasmic and membrane fractions. The western blotting assay was carried out with the indicated antibodies. The quantitative analyses of ANXA2/references (including ANXA2/HistonH3 in the nucleus, ANXA2/ 3 -tubulin in the cytoplasm and ANXA2/atp A1 at the membrane) were shown in the lower panel $(n=3$, SEM). D U2-OS cells stably expressing H125-TRIM21 were treated with DMSO or TET and added with 3-MA (10 nM) to inhibit autophagy as indicated. The living cells were used to observe the location of GFP-ANXA2. E U2-OS cells stably expressing H125-TRIM21 were transfected with si-NC or si-Beclin 1 and then treated with TET for IF assay using the ANXA2 and Beclin 1 antibodies (upper panel). Lower panel: the percentage of cells showing PM translocation of ANXA2 of 60 cells. Upper panel: U2-OS cells stably expressing H125TRIM21 were treated with DMSO or TET to perform IF assay using ANXA2 and LC3 antibodies (F), ANXA2 and CD63 antibodies (G), and ANXA2 and LAMP1 antibodies $(\mathbf{H})$. Percentages of the cells with colocalization of ANXA2 with LC3 or ANXA2 with CD63 of 60 cells were shown in their lower or right panels, respectively. Scale bar: $10 \mu \mathrm{m} .{ }^{*} p<0.05,{ }^{* *} p<0.01,{ }^{* * *} p<0.001$. All data were representative of three independent experiments.

overexpression of TIRM21 upregulated the expression of ANXA2 at the cytoplasm and cell membrane, while decreased its level within the nucleus (Fig. 3C).

According to a recent study, ANXA2 was incorporated into autophagosomes after interferon- $\gamma$ (IFN- $\gamma$ ) stimulation, followed by the fusion with multivesicular bodies (MVBs) for PM, but not with the lysosome ${ }^{30}$. We then investigated whether the trafficking of ANXA2 was related to the autophagy induced by TRIM21. As shown in Fig. 3D, TRIM21 overexpression resulted in obvious trafficking of GFP-ANXA2 to the PM in living cells (arrows). However, the addition of 3-MA, an upstream inhibitor of autophagy, significantly inhibited ANXA2 localization at the PM. Furthermore, knockdown of Beclin 1 significantly decreased the trafficking of ANXA2 to the PM which mediated by the TRIM21 overexpression (Fig. 3E). IF assay further confirmed the colocalization of ANXA2 with LC3 upon TRIM21 overexpression (Fig. 3F), indicating that ANXA2 was incorporated into the autophagosomes. Next, we observed the colocalization of ANXA2 with CD63 (a marker of MVBs) in the TRIM21overexpressed cells; however, no colocalization of ANXA2 and LAMP1 (a lysosomal marker) was observed, indicating that ANXA2, after incorporating into the autophagosomes, was subsequently inserted into the MVBs for PM localization, but not for lysosomes (Fig. 3G, H). These results suggest that the translocation of ANXA2 toward PM is dependent on TRIM21-induced autophagy and this translocation might favor TRIM21-induced autophagy.

\section{Co-expression of TRIM21 and ANXA2 at the PM in OS tissues}

Having established a positive correlation of TRIM21 with the PM localized ANXA2, we next sought to extend this finding into clinical context by using the TMAs of OS tissues. As shown in Fig. 4A, B, 95\% of OS tissues positively expressed TRIM21 and $97.5 \%$ positively expressed ANXA2 at the PM. Moreover, a positive correlation between TRIM21 expression in the whole-cell and ANXA2 expression at the PM was established, with a statistical significance $(R=0.64, \quad P<0.0001$, Fig. 4C). Three representative cases were shown in Fig. 4D.

\section{TRIM21 disrupts the ANXA2-TFEB complex and promotes} the nuclear translocation of TFEB

In lung epithelial cells, ANXA2 has been reported to interact with TFEB, a master transcription factor for autophagy, thus impeding autophagy through cytoplasmic retention and inactivation of $\mathrm{TFEB}^{27,31-33}$. Consistent with this, our co-IP assay did confirm that TFEB interacted with ANXA2 in the U2-OS cells (Fig. 5A). Of significance, overexpression of TRIM21 diminished the interaction between ANXA2 and TFEB, while favoring ANXA2 and TRIM21 interaction (Fig. 5B). A similar result was observed by IF assay, in which overexpression of TRIM21 reduced the colocalization of ANXA2 and TFEB in the cytoplasm (Fig. 5C). Instead, an increase of PM localization of ANXA2 and nuclear localization of TFEB was found (Fig. 5C). Thus, these results collectively suggest that TRIM21 impedes the cytoplasmic association between ANXA2 and TFEB, and that the translocation of ANXA2 towards PM might contribute to the release of TFEB from the complex of ANAX2-TFEB.

We then evaluated the impacts of ANXA2 and TRIM21 on the localization of TFEB. As shown in Fig. 5D, E, either knockdown of ANXA2 or overexpression of TRIM21 promoted the nuclear translocation of TFEB. Similarly, the increased nuclear translocation of TFEB was confirmed using the subcellular fractionation assay upon ANXA2 knockdown and the diminished nuclear translocation of TFEB was found upon TRIM21 knockdown (Fig. 5F, G). These results indicate that TRIM21 promotes the nuclear translocation of TFEB, whereas ANXA2 has the opposite effect. As TFEB can upregulate autophagy-related genes such as $p 62 / S Q S T M 1^{31}$, we explored the role of ANXA2 and TRIM21 in regulating p62 mRNA expression. As shown in Fig. 5H, ANXA2 knockdown caused a significant increase in p62 mRNA expression, while EGFP-ANXA2 overexpression decreased it. Interestingly, we demonstrated that TRIM21 played an opposite role in regulating 


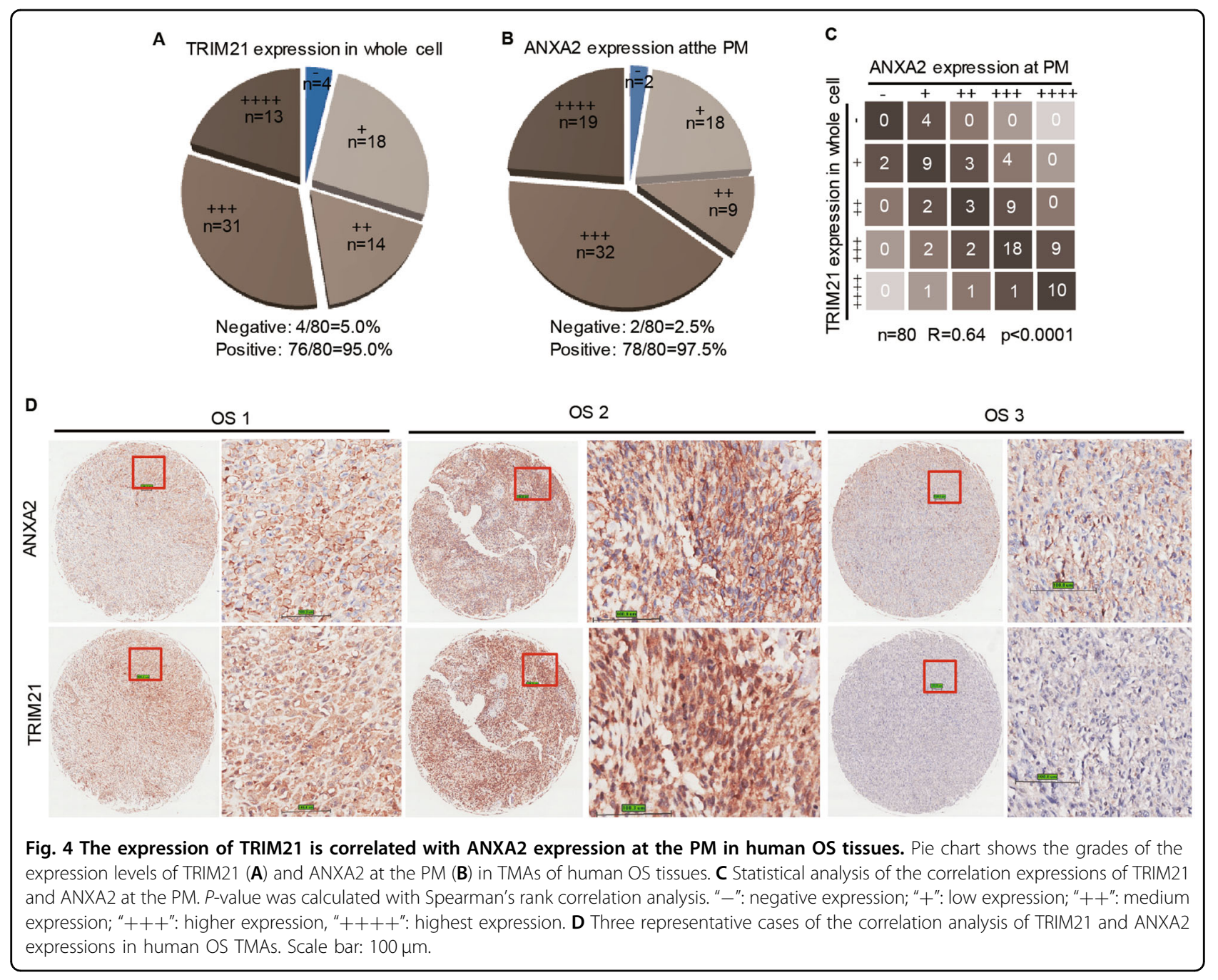

p62 (Fig. 5I). These results indicate that ANXA2 and TRIM21 affect the mRNA expression of p62 through regulating the TFEB translocation. Furthermore, overexpression of EGFP-ANXA2 lessened the nuclear expression of TFEB, whereas overexpression of TRIM21 augmented the nuclear expression of TFEB. This augments of nuclear TFEB was compromised by the overexpression of EGFP-ANXA2 (Fig. 5J, K). These results suggest that ANXA2 suppresses the nuclear translocation of TFEB induced by TRIM21 and thus triggering OS cell autophagy (Fig. 5L).

\section{TRIM21 inhibits osteogenic differentiation of OS cells by inducing autophagy}

Autophagy has been shown to positively regulate osteogenic differentiation of osteoblast $^{34-37}$, we then hypothesized that TRIM21 might regulate OS cell differentiation through the induction of autophagy. Serum starvation caused an increase of LC3-II, accompanied by a downregulation of RUNX2 (Supplementary Fig. S2a, b), a master osteogenic marker for osteoblast and $\mathrm{OS}^{38}$. Conversely, inhibition of autophagy with $\mathrm{CQ}$ elevated the expression of RUNX2, which was re-decreased by the protein synthesis inhibitor CHX (Suppleentary Fig. S2c-f). These results suggested that autophagy might inhibit the osteogenic differentiation of OS cells through a transcriptional regulation rather than autophagy degradation of RUNX2 (Supplementary Fig. S2g). Next, we determined the expression of TRIM21 in a series of OS cells with different differentiation status. It has been reported that the degrees of differentiation in OS cells MG63, U2-OS, Saos-2 were gradually increased ${ }^{39-42}$. In line with this, our results confirmed that the levels of the differentiation markers RUNX2 and ALP were gradually increased in the MG63, U2-OS, and Saos-2 cells (Supplementary Fig. S2h, i). Interestingly, the degrees of autophagy and expression of TRIM21 was gradually decreased in these cells. These results suggest that the expression of TRIM21 is negatively correlated with OS differentiation. 


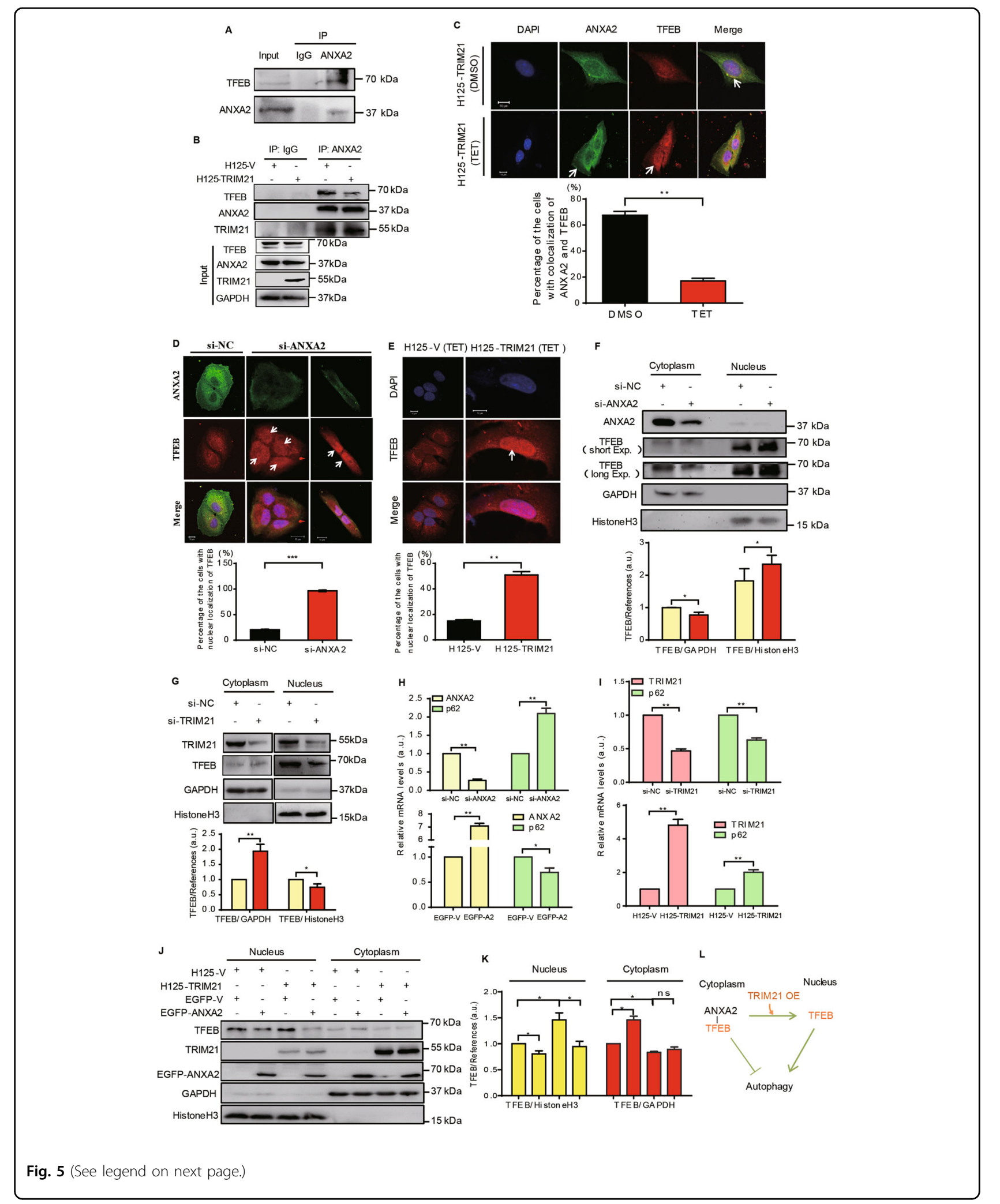

We then explored the implication of TRIM21 in OS differentiation. As shown in Fig. 6A, B, overexpression of HA-TRIMA21 significantly reduced the level of RUNX2, while TRIM21 knockdown increased RUNX2 expression and ALP activity (Fig. 6C-E). Moreover, overexpression of HA-TRIM21 significantly reduced the expression of 


\begin{abstract}
(see figure on previous page)
Fig. 5 TRIM21 separates the ANXA2-TFEB complex and thus facilitates the autophagy in OS cells. A U2-OS cells were used to perform co-IP assay with ANXA2 antibody. B U2-OS cells stably expressing H125-V or H125-TRIM21 were used to perform co-IP assay with IgG and ANXA2 antibodies after TET $(10 \mu \mathrm{g} / \mathrm{ml})$ treatment. C U2-OS cells stably expressing H125-TRIM21 were treated with DMSO or TET and then performed IF using ANXA2 and TFEB antibodies (Upper panel). (Lower panel) Percentage of the cells with colocalization of ANXA2 and TFEB in 50 cells. D U2-OS cells were transfected with si-NC or si-ANXA2 and then performed IF using ANXA2 and TFEB antibodies (upper panel). Lower panel: percentage of the cells with nuclear localization of TFEB in 80 cells. Arrows: nucleus. E U2-OS cells stably expressing H125-TRIM21 or H125-V were treated with TET and then performed IF using the TFEB antibody (Upper panel). Lower panel: Percentage of the cells with nuclear localization of TFEB in 80 cells. Arrow: nucleus. U2-OS cells were transfected with si-ANXA2 (F) or Si-TRIM21 (G) and then performed subcellular fractionation assay to isolate the nuclear and cytoplasmic fractions (Upper panel). The corresponding quantitative analyses of TFEB/References were shown in their lower panels, respectively $(n=$ 3, SEM). H, I U2-OS cells transfected with si-ANXA2, EGFP-ANXA2 or si-TRIM21, or the stable U2-OS cells expressing H125-TRIM21 or H125-V with TET treatment as indicated to perform qRT-PCR assays to analyze the mRNA expression of p62, ANXA2, or TRIM21 $(n=3$, SEM). J U2-OS cells stably expressing H125-TRIM21 or H125-V were transfected with EGFP-ANXA2 as indicated and treated with TET to perform the subcellular fractionation assay using the indicated antibodies. $\mathbf{K}$ The quantitative analyses of TFEB/References in the nucleus and cytoplasm in $\mathbf{J}(n=3$, SEM). $\mathbf{L}$ A supposed association of TRIM21, ANXA2, and TFEB. Scale bar: $10 \mu \mathrm{m} .{ }^{*} p<0.05,{ }^{* *} p<0.01,{ }^{* * *} p<0.001$. All data were representative of three independent experiments.
\end{abstract}

RUNX2 and ALP at the transcriptional levels (Fig. 6F). Inversely, TRIM21 knockdown significantly enhanced the transcriptional levels of RUNX2 and ALP (Fig. 6G). Thus, TRIM21 inhibits OS cell differentiation marked with the negative regulation of the transcriptional expressions of RUNX2 and ALP.

To examine whether the effect of TRIM21 on OS cell differentiation was dependent on the TRIM21-induced autophagy, the autophagy inhibitor CQ was applied. As shown in Fig. $6 \mathrm{H}$, overexpression of HA-TRM21 diminished the level of RUNX2; however, it was restored upon the treatment of $\mathrm{CQ}$, but not the proteasome inhibitor, MG132 (Fig. 6H). Similarly, the decline of ALP activity induced by TRIM21 overexpression was also restored by the CQ (Fig. 6I). Collectively, these results indicate that autophagy is a prerequisite for the inhibitory role of TRIM21 in OS cell differentiation (Fig. 6J). Next, we tested whether ANXA2 was involved in the regulation of OS cell differentiation mediated by TRIM21. In agreement with the previous report ${ }^{25}$, knockdown of ANXA2 decreased RUNX2 expression (Fig. 6K), while overexpression of ANXA2 increased its expression level, which was suppressed by the autophagy activator RAPA (Fig. 6L). Furthermore, ANXA2 overexpression reversed the downregulated trend of RUNX2 induced by TRIM21 overexpression (Fig. 6M), suggesting that ANXA2 counteracted TRIM21-mediated differentiation inhibition. In addition, TFEB knockdown compromised the decreasing trend of RUNX2 induced by TRIM21 overexpression (Supplementary Fig. S3), indicating that TFEB plays a role in TRIM21-regulated RUNX2 expression.

\section{Discussion}

Recently, TRIM21, despite its role as E3 ubiquitin ligases, has been extensively demonstrated to be involved in the regulation of autophagy ${ }^{7,29}$, yet its role in OS cell autophagy remains unclear. In this study, we showed that TRIM21 promoted OS cell autophagy by enhancing the autophagic flux and the degradation of p62 (Fig. 1). A previous study has reported that TRIM21 ubiquitylates p62 and abrogates p62 oligomerization and sequestration of client proteins for autophagic degradation under proteotoxic stress and starvation ${ }^{43}$. In other cases, TRIM21 has been shown to facilitate autophagy by assembling core components of autophagic machinery including ULK1, BECN1, and p62 in the cells responding to IFN- $\gamma$ stimulation $^{6,44}$. Thus, both the influence of TRIM21 on autophagy and the interplay between TRIM21 and p62 are highly cell-type dependent. ANXA2 was screened and identified as an interesting interaction partner of TRIM21 (Fig. 2) due to its backgrounds in both autophagy and osteoblast differentiation $^{25-28}$. ANXA2 has been demonstrated to not only positively regulate autophagy ${ }^{45-47}$ but also participate in the negative regulation of autophagy by impeding autophagic flux and inducing cytoplasmic retention of $\mathrm{TFEB}^{27}$. TFEB upregulates the number of autophagosomes and increases gene expressions of ATG9B, p62/SQSTM1, LC3, UVRAG, WIPI, VPS11, and VPS18, the direct targets of TFEB ${ }^{31}$. Here we showed that ANXA2 not only inhibited OS cell autophagy but also antagonized TRIM21-induced autophagy (Fig. 2). Overexpression of TRIM21 partly compromised the inhibitory role of ANXA2 in autophagy through disrupting the ANXA2-TFEB complex, abolishing the cytoplasmic retention of TFEB, and facilitating the nuclear translocation of TFEB (Figs. 2 and 5). Collectively, these results suggest that the TRIM21/ANXA2/TFEB axis is involved in OS cell autophagy (Fig. 7).

The relationships between autophagy and differentiation are still controversial. During osteogenesis and osteoclastogenesis, autophagy is actively induced and required for differentiation ${ }^{34,35,37,48-51}$. Other research showed that pneumolysin (PLY), a major virulence factor of Streptococcus pneumonia, inhibits osteoblast differentiation by inducing autophagy ${ }^{39}$. Mechanistically, PLY-induced autophagy downregulates the expression 


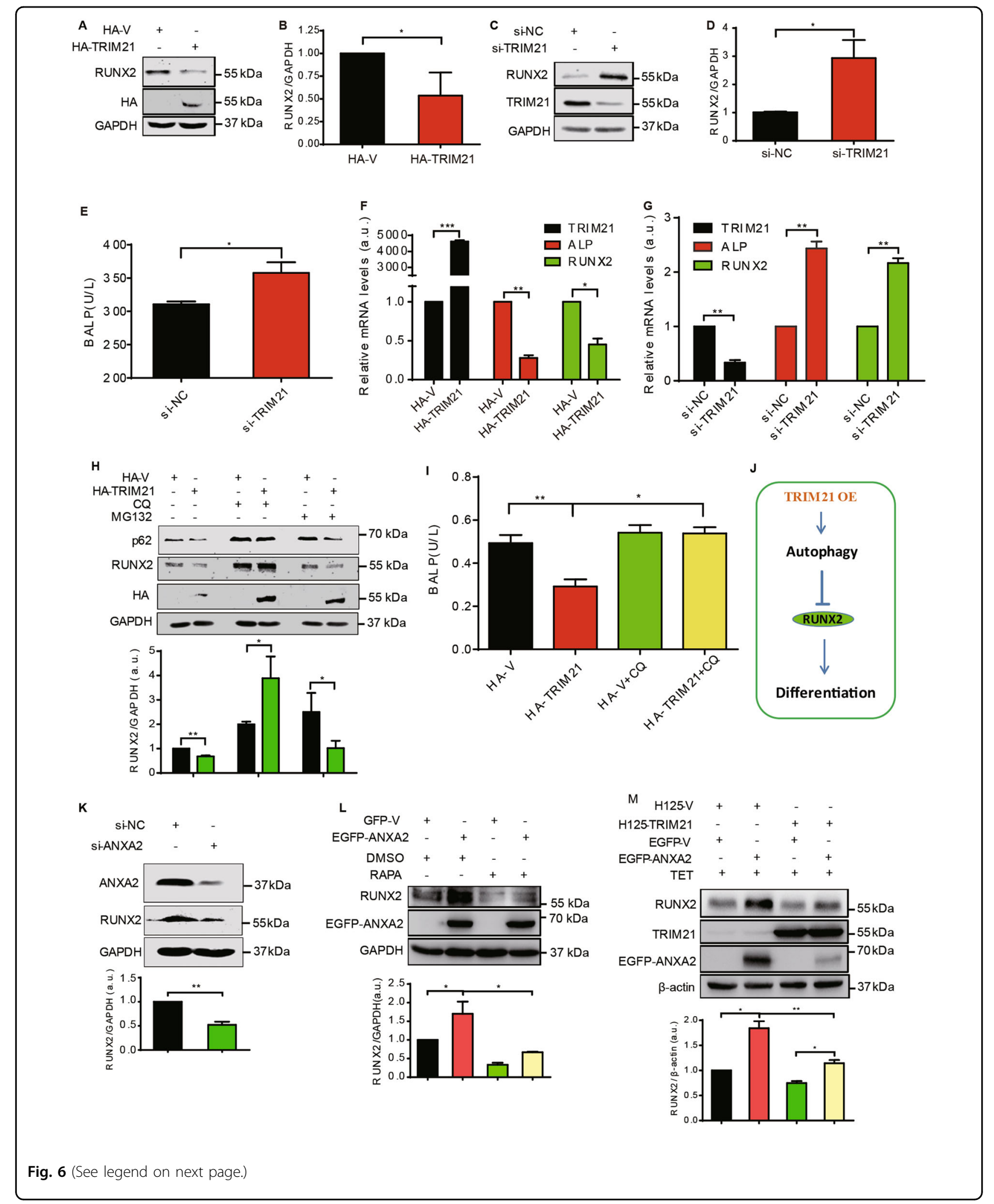

of transcription factor Sp1, essential for differentiation, and subsequently inhibits osteoblast differentiation ${ }^{39}$. Sp1 upregulates RUNX2 and both of them are key transcription factors of osteoblast differentiation ${ }^{52}$. Here, TRIM21-induced autophagy inhibited the expression of RUNX2 (Fig. 6). It is likely that the 
(see figure on previous page)

Fig. 6 TRIM21 inhibits OS cell differentiation in an autophagy-dependent manner. A-D OS cells were transfected with HA-TRIM21 (A) or siRNA of TRIM21 (C) and performed western blotting assay. The corresponding quantitative analyses of the RUNX2/GAPDH ratio were shown in B and $\mathbf{D}(n$ $=3$, SEM). E U2-OS cells were transfected with siRNA of TRIM21 and harvested for detection of ALP activity using ELISA assay ( $n=3$, SEM). U2-OS cells were transfected with HA-TRIM21 (F) or TRIM21 siRNA (G), respectively, and collected for detection of the expression of TRIM21, ALP, and RUNX2 by qRT-PCR assay $(n=3$, SEM). H U2-OS cells were transfected with HA-TRIM21 and treated with CQ and MG132 (100 nM) for $24 \mathrm{~h}$. The cells were harvested and performed western blotting assay with the indicated antibodies. The ratio of RUNX2/GAPDH was shown in its lower panel $(n=3$, SEM). I U2-OS cells were transfected with HA-TRIM21 or HA-vector and harvested for detection of ALP activity using ELISA assay $(n=3$, SEM). J A model of the relationship between TRIM21 and differentiation. TRIM21 OE: TRIM21 overexpression. $\mathbf{K}$ OS cells transfected with si-NC or si-ANXA2 were performed western blotting assay (Upper panel). RUNX2/GAPDH was shown in its lower panel $(n=3$, SEM). L U2-OS cells were transfected with EGFP-V or EGFP-ANXA2 and treated with RAPA $(1.5 \mu \mathrm{M}, 24 \mathrm{~h}$ ) as indicated to perform western blotting assay (upper panel). Lower panel: the ratio of RUNX2/GAPDH ( $n=3$, SEM). M U2-OS cells stably expressing H125-TRIM21 or H125-V were transfected with EGFP-ANXA2 or EGFP-vector and treated with TET as indicated to perform western blotting assay. RUNX2/ $\beta$-actin was shown in its lower panel $\left(n=3\right.$, SEM). ${ }^{*} p<0.05$; ${ }^{* *} p<0.01$. All data were representative of three independent experiments.

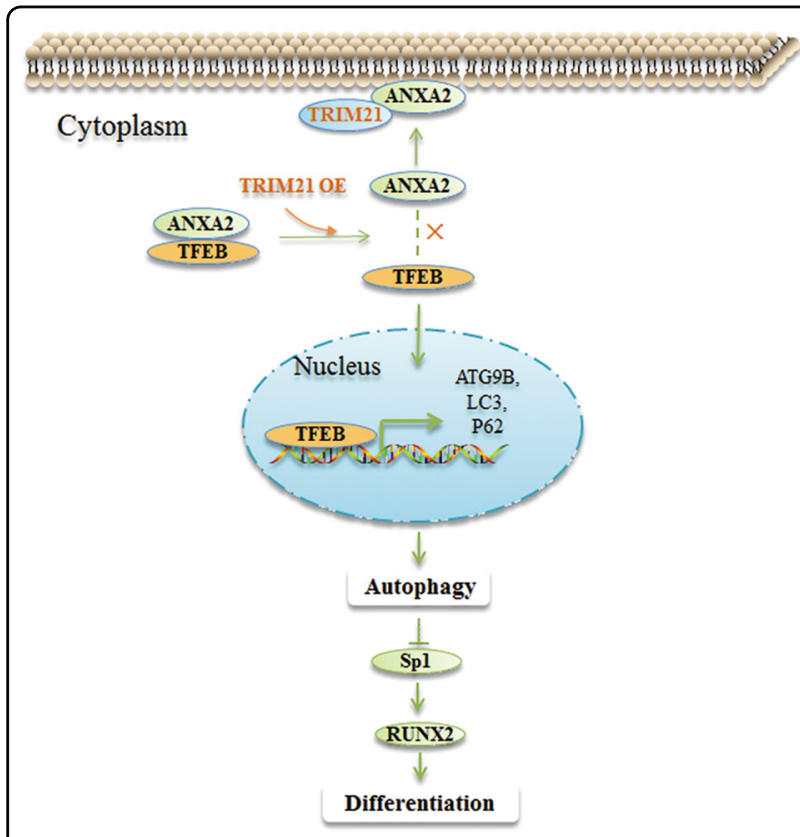

Fig. 7 A schematic model of TRIM21 regulating OS autophagy and differentiation by interacting with ANXA2. When TRIM21 is overexpressed in OS cells, it competitively binds to ANXA2 and thus facilitating the translocation of ANXA2 toward PM; this enables the disassociation of TFEB from the ANXA2-TFEB complex, allowing TFEB entering into the nucleus. Nuclear TFEB promotes the transcription of autophagy-related genes, including $A T G 9 B, L C 3$, and P62, and inhibits OS differentiation, presumably, through regulating the Sp1/ RUNX2 signaling in OS cells. TRIM21 OE: TRIM21 overexpression. $x$ : disassociation of ANXA2-TFEB complex.

inhibitory effect of autophagy induced by TRIM21 on the differentiation is through the Sp1/RUNX2 signaling in OS cells (Fig. 7).

ANXA2 has been reported to be implicated in the positive regulation of osteoblastic differentiation ${ }^{25,26,28,53}$. Consistently, our result showed that ANXA2 positively regulated OS differentiation (Fig. 6). ANXA2 is reported to express in the cytoplasm, nucleus, and $\mathrm{PM}^{54}$, and able to translocate toward PM under several conditions ${ }^{55-57}$. Herein, we demonstrated that TRIM21 overexpression facilitated the translocation of ANXA2 toward PM (Fig. 3), which might be beneficial for the release of TFEB from its inhibitory complex and thus activating cell autophagy (Fig. 7).

In conclusion, our study provides deep insights into the biological function and molecular mechanism of TRIM21 in OS autophagy and differentiation, which highlights the potential of TRIM21 silencing as a promising therapeutic strategy for OS. In addition, our findings that the TRIM21/ANXA2/TFEB axis is involved in OS cell autophagy and subsequent differentiation, also might lead to a new clue for OS treatment.

\section{Acknowledgements}

We thank Han's Lab of Xiamen University for providing us with the cDNA of human TRIM21 and ANXA2.

\section{Author details \\ ${ }^{1}$ Key Laboratory of Functional Protein Research of Guangdong Higher Education Institutes and MOE Key Laboratory of Tumor Molecular Biology, Institute of Life and Health Engineering, Jinan University, Guangzhou 510632, China. ${ }^{2}$ Department of Bone and Joint Surgery, Institute of Orthopedic Diseases, The First Affiliated Hospital, Jinan University, Guangzhou, Guangdong 510630, China. ${ }^{3}$ Department of Pathology, Henan Provincial People's Hospital, People's Hospital of Zhengzhou University, 7 Weiwu Road, Zhengzhou 450003, China. ${ }^{4}$ Center of Kidney Disease, Huadu District People's Hospital, Southern Medical University, Guangzhou, Guangdong 510800, China}

\section{Author contributions}

Z.H.T. designed experiments, analyzed the data, drafted and revised the manuscript. Z.Q. and W.B. performed most of the experiments. L.J., T.K.L., L.J., X.L., Y.J., L.X., and L.Q. performed some of the experiments. L.Q.Y. and Z.Y.F. performed some of the experiments and analyzed the data. L.W. analyzed the data. L.L. analyzed the data and revised the manuscript. G.X. designed experiments, analyzed the data, drafted the manuscript, and approved the final version to be published.

\section{Ethics}

Tissue microarrays: The expressions of TRIM21 and ANXA2 were analyzed using two consecutively numbered commercial tissue microarrays (TMAs) of osteosarcoma (OS804C, Alenabio, Xi'an, China), which includes 80 sections of OS tissue samples and every 2 sections coming from one OS case. The company stated that all human tissues are collected under IRB (Institutional Review Board) and HIPPA-approved protocols. 


\section{Funding}

This work was supported by the Natural Science Foundation of Guangdong Province (2018A030313544, 2019A1515011082, 2019A1515010182, and 2017A030313665), the National Natural Science Foundation of China (81602360, 81800675, and 82072470), the Guangzhou Science and Technology Project (201707010263 and 201804010066), the Macau Foundation for Development of Science and Technology (0029/2019/A), and Youth Talent Support Project of Guangzhou Association For Science and Technology (X20200301018)

\section{Conflict of interest}

The authors declare that they have no conflict of interest.

\section{Publisher's note}

Springer Nature remains neutral with regard to jurisdictional claims in published maps and institutional affiliations.

Supplementary Information accompanies this paper at (https://doi.org/ 10.1038/s41419-020-03364-2).

Received: 27 April 2020 Revised: 15 December 2020 Accepted: 18 December 2020

Published online: 06 January 2021

\section{References}

1. Lewis, I. J. et al. Improvement in histologic response but not survival in osteosarcoma patients treated with intensified chemotherapy: a randomized phase III trial of the European Osteosarcoma Intergroup. J. Nat/ Cancer Inst. 99 112-128 (2007).

2. Lamoureux, F. et al. Selective inhibition of BET bromodomain epigenetic signalling interferes with the bone-associated tumour vicious cycle. Nat. Commun. 5, 3511 (2014).

3. Camuzard, O., Santucci-Darmanin, S., Carle, G. F. \& Pierrefite-Carle, V. Role of autophagy in osteosarcoma. J. Bone Oncol. 16, 100235 (2019).

4. Kansara, M. \& Thomas, D. M. Molecular pathogenesis of osteosarcoma. DNA Cell Biol. 26, 1-18 (2007).

5. Lamplot, J. D. et al. The current and future therapies for human osteosarcoma. Curr. Cancer Ther. Rev. 9, 55-77 (2013).

6. Di Rienzo, M., Romagnoli, A., Antonioli, M., Piacentini, M. \& Fimia, G. M. TRIM proteins in autophagy: selective sensors in cell damage and innate immune responses. Cell Death Differ. 27, 887-902 (2020).

7. Kimura, T. et al. TRIM-directed selective autophagy regulates immune activation. Autophagy 13, 989-990 (2017).

8. Kunishita, Y. et al. TRIM21 dysfunction enhances aberrant B-cell differentiation in autoimmune pathogenesis. Front Immunol. 11, 98 (2020).

9. Zhou, G. et al. Tripartite motif-containing (TRIM) 21 negatively regulates intestinal mucosal inflammation through inhibiting $\mathrm{TH} 1 / \mathrm{TH} 17$ cell differentiation in patients with inflammatory bowel diseases. J. Allergy Clin. Immunol. 142, 1218-1228. e1212 (2018).

10. Ding, Q. et al. Downregulation of TRIM21 contributes to hepatocellular carcinoma carcinogenesis and indicates poor prognosis of cancers. Tumour Biol. 36, 8761-8772 (2015).

11. Zhou, W. et al. Decreased expression of TRIM21 indicates unfavorable outcome and promotes cell growth in breast cancer. Cancer Manag. Res. 10, 3687-3696 (2018).

12. Jin, Y. et al. TRIM21 mediates ubiquitination of Snail and modulates epithelial to mesenchymal transition in breast cancer cells. Int. J. Biol. Macromol. 124, 846-853 (2019).

13. Brauner, S. et al. Reduced expression of TRIM21/Ro52 predicts poor prognosis in diffuse large B-cell lymphoma patients with and without rheumatic disease. J. Intern. Med. 278, 323-332 (2015).

14. Nguyen, J. Q. \& Irby, R. B. TRIM21 is a novel regulator of Par-4 in colon and pancreatic cancer cells. Cancer Biol. Ther. 18, 16-25 (2017).

15. Zeng, Q. Z. et al. MWHAZ binds to TRIM21 but is not involved in TRIM21stimulated osteosarcoma cell proliferation. Biomed. Environ. Sci. 31, 186-196 (2018).

16. Li, Y. H. et al. PRMT5-TRIM21 interaction regulates the senescence of osteosarcoma cells by targeting the TXNIP/p21 axis. Aging 12, 2507-2529 (2020).
17. Liang, G. H. et al. Transcriptional regulation of Runx2 by HSP90 controls osteosarcoma apoptosis via the AKT/GSK-3beta/beta-catenin signaling. J. Cell. Biochem. 119, 948-959 (2018).

18. Gao, X. et al. hnRNPK inhibits GSK3beta Ser9 phosphorylation, thereby stabilizing C-FLIP and contributes to TRAlL resistance in H1299 lung adenocarcinoma cells. Sci. Rep. 6, 22999 (2016).

19. Gao, X. et al. 14-3-3zeta reduces DNA damage by interacting with and stabilizing proliferating cell nuclear antigen. J. Cell. Biochem. 116, 158-169 (2015).

20. Huang, W. S. et al. ERK1/2-mediated cytoplasmic accumulation of hnRNPK antagonizes TRAlL-induced apoptosis through upregulation of XIAP in H1299 cells. Biomed. Environ. Sci. 30, 473-481 (2017).

21. Gao, X., Xing, D., Liu, L. \& Tang, Y. H-Ras and PI3K are required for the formation of circular dorsal ruffles induced by low-power laser irradiation. J. Cell. Physiol. 219, 535-543 (2009).

22. Mauthe, $M$. et al. Chloroquine inhibits autophagic flux by decreasing autophagosome-lysosome fusion. Autophagy 14, 1435-1455 (2018).

23. Wang, Y. et al. Isodeoxyelephantopin induces protective autophagy in lung cancer cells via Nrf2-p62-keap1 feedback loop. Cell Death Dis. 8, e2876 (2017).

24. Khaminets, A., Behl, C. \& Dikic, I. Ubiquitin-dependent and independent signals in selective autophagy. Trends Cell Biol. 26, 6-16 (2016)

25. Genetos, D. C., Wong, A., Weber, T. J., Karin, N. J. \& Yellowley, C. E. Impaired osteoblast differentiation in annexin A2- and -A5-deficient cells. PLOS ONE $\mathbf{9}$ e107482 (2014)

26. Gillette, J. M. \& Nielsen-Preiss, S. M. The role of annexin 2 in osteoblastic mineralization. J. Cell Sci. 117, 441-449 (2004).

27. Wang, K. et al. Identification of ANXA2 (annexin A2) as a specific bleomycin target to induce pulmonary fibrosis by impeding TFEB-mediated autophagic flux. Autophagy 14, 269-282 (2018).

28. Gillette, J. M., Chan, D. C. \& Nielsen-Preiss, S. M. Annexin 2 expression is reduced in human osteosarcoma metastases. J. Cell. Biochem. 92, 820-832 (2004).

29. Reddy, B. A. et al. Nucleotide biosynthetic enzyme GMP synthase is a TRIM21controlled relay of p53 stabilization. Mol. Cell 53, 458-470 (2014).

30. Chen, Y. D. et al. Exophagy of annexin A2 via RAB11, RAB8A and RAB27A in IFN-gamma-stimulated lung epithelial cells. Sci. Rep. 7, 5676 (2017).

31. Settembre, C. et al. TFEB links autophagy to lysosomal biogenesis. Science $\mathbf{3 3 2}$, 1429-1433 (2011).

32. Mammucari, $\mathrm{C}$. et al. FoxO3 controls autophagy in skeletal muscle in vivo. Cell Metab. 6, 458-471 (2007).

33. Martina, J. A. et al. The nutrient-responsive transcription factor TFE3 promotes autophagy, lysosomal biogenesis, and clearance of cellular debris. Sci. Signal. 7 ra9 (2014).

34. Mizushima, N. \& Levine, B. Autophagy in mammalian development and differentiation. Nat. Cell Biol. 12, 823-830 (2010).

35. Pantovic, A. et al. Coordinated time-dependent modulation of AMPK/Akt/ mTOR signaling and autophagy controls osteogenic differentiation of human mesenchymal stem cells. Bone 52, 524-531 (2013).

36. Green, D. R. \& Levine, B. To be or not to be? How selective autophagy and cell death govern cell fate. Cell 157, 65-75 (2014).

37. Chen, W. T., Zhang, F., Zhao, X. Q., Yu, B. \& Wang, B. W. Galectin-3 and TRIM16 coregulate osteogenic differentiation of human bone marrow-derived mesenchymal stem cells at least partly via enhancing autophagy. Bone 131, 115059 (2019).

38. Geoffroy, V., Kneissel, M., Fournier, B., Boyde, A. \& Matthias, P. High bone resorption in adult aging transgenic mice overexpressing cbfa1/runx2 in cells of the osteoblastic lineage. Mol. Cell. Biol. 22, 6222-6233 (2002).

39. Kim, J., Lee, H. W., Rhee, D. K., Paton, J. C. \& Pyo, S. Pneumolysin-induced autophagy contributes to inhibition of osteoblast differentiation through downregulation of Sp1 in human osteosarcoma cells. Biochim. Biophys. Acta Gen. Subj. 1861, 2663-2673 (2017).

40. Wang, W. et al. The role of the Wnt/beta-catenin pathway in the effect of implant topography on MG63 differentiation. Biomaterials 33, 7993-8002 (2012).

41. Reffitt, D. M. et al. Orthosilicic acid stimulates collagen type 1 synthesis and osteoblastic differentiation in human osteoblast-like cells in vitro. Bone $\mathbf{3 2}$, 127-135 (2003).

42. Luo, X. et al. Osteogenic BMPs promote tumor growth of human osteosarcomas that harbor differentiation defects. Lab. Invest. 88, 1264-1277 (2008). 
43. Pan, J. A. et al. TRIM21 ubiquitylates SQSTM1/p62 and suppresses protein sequestration to regulate redox homeostasis. Mol. Cell 62, 149-151 (2016).

44. Kimura, T. et al. TRIM-mediated precision autophagy targets cytoplasmic regulators of innate immunity. J. Cell Biol. 210, 973-989 (2015).

45. Morozova, K. et al. Annexin A2 promotes phagophore assembly by enhancing Atg16L(+) vesicle biogenesis and homotypic fusion. Nat. Commun. 6, 5856 (2015).

46. Moreau, K. et al. Transcriptional regulation of Annexin A2 promotes starvationinduced autophagy. Nat. Commun. 6, 8045 (2015).

47. Li, R. et al. Annexin A2 regulates autophagy in Pseudomonas aeruginosa infection through the Akt1-mTOR-ULK1/2 signaling pathway. J. Immunol. 195, 3901-3911 (2015).

48. Tao, Z., Liu, L., Zheng, L. D. \& Cheng, Z. Autophagy in adipocyte differentiation. Methods Mol. Biol. 1854, 45-53 (2019).

49. Riffelmacher, T., Richter, F. C. \& Simon, A. K. Autophagy dictates metabolism and differentiation of inflammatory immune cells. Autophagy 14, 199-206 (2018).

50. Wan, Y., Zhuo, N., Li, Y., Zhao, W. \& Jiang, D. Autophagy promotes osteogenic differentiation of human bone marrow mesenchymal stem cell derived from osteoporotic vertebrae. Biochem. Biophys. Res. Commun. 488, 46-52 (2017).
51. Qi, M. et al. Autophagy maintains the function of bone marrow mesenchymal stem cells to prevent estrogen deficiency-induced osteoporosis. Theranostics 7, 4498-4516 (2017).

52. Zhang, Y. et al. Co-stimulation of the bone-related Runx2 P1 promoter in mesenchymal cells by SP1 and ETS transcription factors at polymorphic purine-rich DNA sequences (Y-repeats). J. Biol. Chem. 284, 3125-3135 (2009).

53. Davies, O. G. et al. Osteoblast-derived vesicle protein content is temporally regulated during osteogenesis: implications for regenerative therapies. Front. Bioeng. Biotechnol. 7, 92 (2019).

54. Waisman, D. M. Annexin II tetramer: structure and function. Mol. Cell. Biochem. 149-150, 301-322 (1995).

55. Deora, A. B., Kreitzer, G., Jacovina, A. T. \& Hajjar, K. A. An annexin 2 phosphorylation switch mediates p11-dependent translocation of annexin 2 to the cell surface. J. Biol. Chem. 279, 43411-43418 (2004).

56. Lei, H., Romeo, G. \& Kazlauskas, A. Heat shock protein 90alpha-dependent translocation of annexin $\|$ to the surface of endothelial cells modulates plasmin activity in the diabetic rat aorta. Circ. Res. 94, 902-909 (2004).

57. Li, H., Wang, Y., Lu, Y. \& Li, F. Annexin A2 interacting with ELMO1 regulates HCC chemotaxis and metastasis. Life Sci. 222, 168-174 (2019). 